\title{
A novel CMOS Hall effect sensor
}

\author{
D.S. Mellet ${ }^{a}$, M. du Plessis ${ }^{b}$ \\ ${ }^{a}$ Azoteq Pty(Ltd), 109 Main Street, 7646 Paarl, South Africa \\ ${ }^{\mathrm{b}}$ University of Pretoria, Carl and Emily Fuchs Institute for Microelectronics, 0002 Pretoria, South Africa
}

\begin{abstract}
This paper reports on a new technique for sensing the Hall effect in an integrated CMOS device. Contrary to traditional Hall plates where sensor contacts comprise of highly doped, low ohmic contacts, the proposed sensor makes use of a parasitic vertical pnp bipolar junction transistor (BJT) to sense and amplify the Hall current caused by the Lorentz force in the presence of a perpendicular magnetic field. The Hall effect appears as a current through the emitter of the BJT. The BJT forward gain implies a direct gain of at least $\beta+1$ in the measured signal in comparison to traditional methods.
\end{abstract}

\section{Keywords}

Hall effect, CMOS, Hall current

\section{Introduction}

The state of the art method of sensing the Hall effect in Hall plate devices is through the use of highly doped low ohmic contacts $[1,2,3,4,5,6,7,8]$. This method of sensing is primarily associated with the Hall voltage which in silicon can be very limited in magnitude. A typical silicon Hall plate has a sensitivity of $0.07 \mathrm{~T}^{-1}$ for both voltage mode and current mode of operation [9], and since the Hall voltage scales linearly with the applied DC voltage bias and magnetic field, this relates to a typical signal in the order of $25 \mathrm{mV}$ at a DC bias of $1.7 \mathrm{~V}$ and a magnetic field of $200 \mathrm{mT}$. Taking the geometrical factor into account for a square plate, this signal can diminish further by up to $30 \%$ in magnitude [9]. Replacing the $\mathrm{n}+$ sense contacts in the $\mathrm{n}$-well with $\mathrm{p}$-type implants results in a parasitic pnp BJT formed vertically downward to the substrate through which the Hall current can be effectively measured, very similar to the BJT's used in a CMOS bandgap circuit [10]. This allows for a very simple measuring technique with built-in gain when compared to complex traditional amplification such as instrumentation amplifiers. . As CMOS technologies scale down to smaller geometries, combined with the high biasing voltages and currents required to maximise Hall sensitivities, traditional Hall plates are progressing toward the velocity saturation limits and increased Joule heating. For large geometries this is normally no problem but for $\mu$-Hall devices this becomes a limiting factor [11]. For silicon velocity saturation occurs at approximately $30 \mathrm{kVcm}^{-1}$ and this corresponds to a minimum length of $1.5 \mu \mathrm{m}$ for a voltage bias of $5 \mathrm{~V}$ [9]. In contrast with traditional sensing techniques which require high supply voltages, the technique is capable of attaining a wide dynamic range at very low supply voltages and hence can be applied in low power devices.

Our novel sensing circuit will induce substrate currents through the collector terminals of the vertical pnp BJT's and may require well-known layout techniques to avoid undesired latch up effects. Transistor mismatches, especially pnp transistor beta mismatches on chip, may require DC offset trimming techniques similar to that used in operational amplifier input stage designs. 
This paper begins with a brief summary of the Hall effect physics applicable to the proposed design after which the Hall plate design, layout, experimental procedure and results are explained. The effect of noise and other limitations are then discussed followed by the concluding remarks and suggestions for further research on the topic.

\section{The Hall effect}

\subsection{Hall voltage in long and short geometries}

Fig. 1 illustrates a typical a) long and b) short Hall plate with dimensions length $=l$, width $=w$ and thickness $=t$ and four contacts at its boundaries, with $I>>w$ for the long device and $I<<w$ for the short device. The long geometry in a) is very similar to the $2 \times 9 \mathrm{~cm}$ sample used by Hall in his original experiment [12].
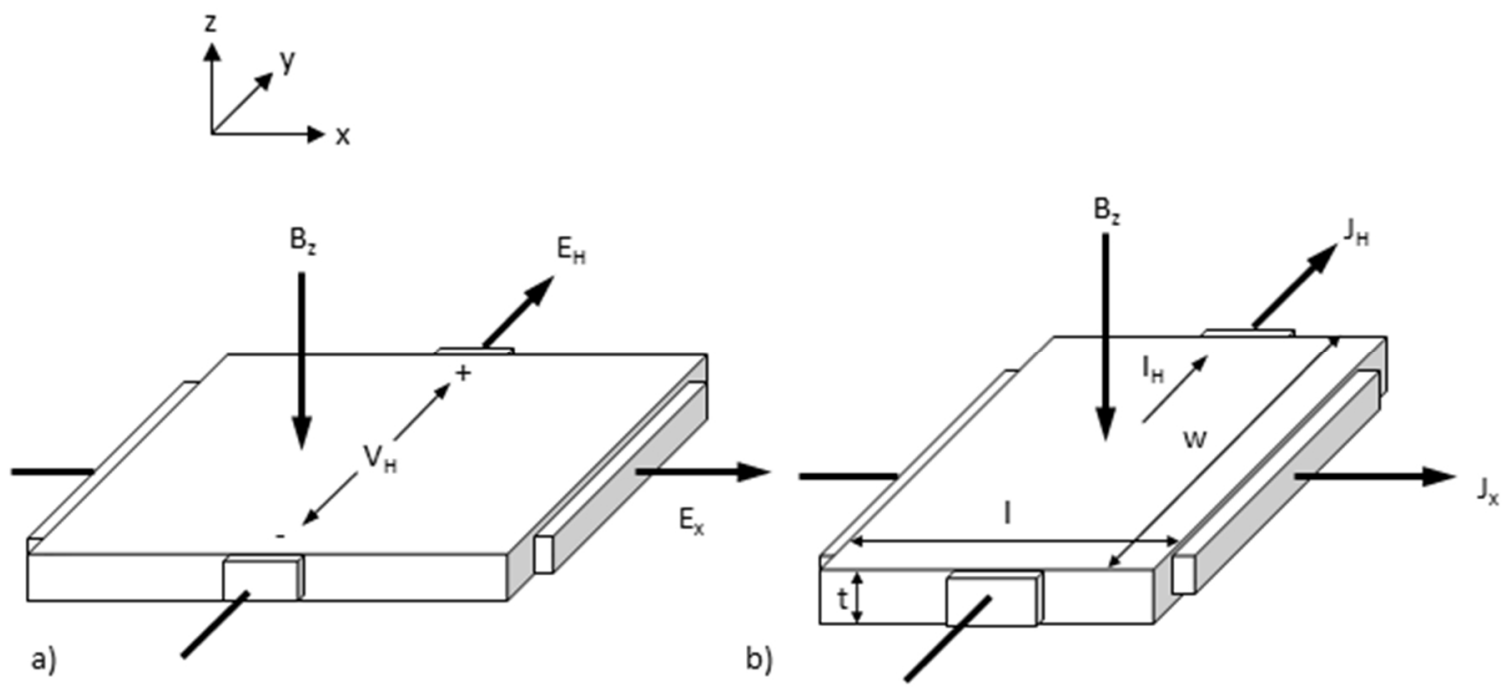

Fig. 1 Hall effect in a) long and b) short samples with applied electric field $E_{x}$ or current density vector $J_{x}$, Hall electric field $E_{H}$ or Hall current density vector $J_{H}$, magnetic field $B_{z}$, width $w$, length $I$, thickness $t$ with resultant a) Hall voltage $V_{H}$ or b) Hall current $I_{H}$

The Hall voltage and current deflection illustrated in Fig. 1 is a direct result of the Lorentz force. A Hall voltage occurs due to the boundaries of the long sample constraining the current flow down the length whilst in the short sample current deflection occurs as the current exits the sample without any boundary interaction. The resulting electric field opposing this force is given [9] by:

$$
\boldsymbol{E}_{H}=\mu\left[\boldsymbol{E}_{x} \times \boldsymbol{B}_{z}\right]
$$


where $\boldsymbol{E}_{H}$ denotes the Hall electric field, $\boldsymbol{\mu}$ the majority charge carrier mobility, $\boldsymbol{E}_{x}$ the externally applied electric field resulting from the voltage bias and $\boldsymbol{B}_{z}$, the perpendicular magnetic induction.

The Hall voltage $V_{H}$ for a constant voltage bias in the long sample in Fig. 1 a) is given by [9]:

$$
V_{H}=\mu \frac{w}{l} G_{H} V_{\text {bias }} B_{\perp}
$$

with $G_{H}$ the geometrical correction factor, $V_{\text {bias }}$ the voltage supplied to the plate and $B_{\perp}$ the perpendicular magnetic field. It can be seen from Eq. ( 2 ) that the Hall voltage is proportional to the voltage supplied as well as the intensity of the perpendicular magnetic field. Similarly then, the Hall current $I_{H}$ for the short sample in Fig. 1 b) can be written [9] as:

$$
I_{H}=\mu \frac{w}{l} G_{H} I_{b i a s} B_{\perp}
$$

and describes the transverse current due to the Hall effect with $I_{\text {bias }}$ the Hall plate biasing current.

\subsection{Offset}

Probably one of the most studied Hall device characteristics is the offset usually described in terms of the offset Hall voltage $V_{\text {off }}$ or offset Hall current $I_{\text {off, }}$ or expressed in terms of an equivalent magnetic field offset $B_{\text {off. }} B_{\text {off }}$ can be derived from the offset voltage or current in terms of the absolute sensitivity $S_{A}$ with units $\mathrm{VT}^{-1}$ for a voltage mode operation and $\mathrm{AT}^{-1}$ for current mode operation as shown in ( 4 ):

$$
B_{o f f}=\frac{V_{o f f}}{s_{A}} \quad \text { and } \quad B_{o f f}=\frac{I_{o f f}}{S_{A}} .
$$

The offset is one of the major deviations observed in a Hall device and is the DC signal measured in the absence of a magnetic field and thus cannot be distinguished from the quasi-static signal of interest [9]. Typically the offset as well as the offset drift of a conventional Hall plate is somewhat larger than the Earth's magnetic field [13]. The main causes for the appearance of an offset are process gradients across the chip, misalignment of masks and/or mechanical stresses resulting from fabrication as well as packaging $[14,15,16,17]$ and can also vary due to supply voltage and temperature [18]. Whereas absolute sensitivity $S_{A}$ above describes how much the output of a sensor will change given a specific magnetic field input, the relative sensitivity is defined as the ratio between the absolute sensitivity and a bias quantity for the Hall voltage and current mode and is given [9] by: 


$$
S_{A V}=\mu \frac{w}{l} G_{H} V_{\text {bias }} \quad \text { and } \quad S_{A I}=\mu \frac{w}{l} G_{H} I_{\text {bias }} .
$$

Substituting ( 5 ) into ( 4 ) shows that the higher the mobility, the lower the equivalent offset. For a typical Hall plate in silicon with $I / w \approx 1$, an offset in the range of $B_{\text {off }} \approx 10 \mathrm{mT}$ can be expected [19].

\subsection{Geometrical correction factor}

The geometrical factor for a point contact rectangular Hall device can be approximated [9] by:

$$
G_{H} \cong 1-\frac{16}{\pi^{2}} e^{\left(-\frac{\pi l}{2 w}\right)}\left[1-\frac{8}{9} e^{\left(-\frac{\pi l}{2 w}\right)}\right]\left(1-\frac{\theta_{H}^{2}}{3}\right)
$$

Eq. ( 6 ) is true for $0.85 \leq I / w \leq \infty$ and $0 \leq \theta_{H} \leq 0.45$ with $\theta_{H}$ in radians. The Hall angle $\theta_{H}$ is given [9] by:

$$
\tan \theta_{H}=\mu B
$$

\section{Design}

Fig. 2 illustrates a cross section and top view of an n-well CMOS Hall plate. The cross section is shown midway along the length of the device. The vertical BJT forming between the p-type contacts in the n-well, through the $n$-well to the substrate can clearly be visualized. As can be seen in the figure, the base of the vertical pnp transistor is formed by the $n$-well and can be contacted by $n+$ contacts. Furthermore, all the vertical pnp transistors share a common collector, namely the $p$-type substrate.

It should be noted that a substrate current will flow through resistors $R_{c 1}$ and $R_{c 2}$, and in order to prevent any problems like latch-up to occur as a result of voltages generated within the substrate, wide $\mathrm{p}+$ substrate contacts should be placed as close to the $\mathrm{p}+$ emitters as possible, as shown in the top view of the figure. It is possible to bias the Hall device such that the BJT becomes forward biased in the active region and in doing so, activate the inherent current gain of the transistor to amplify the Hall current into a useful signal via the emitters of the BJT. 


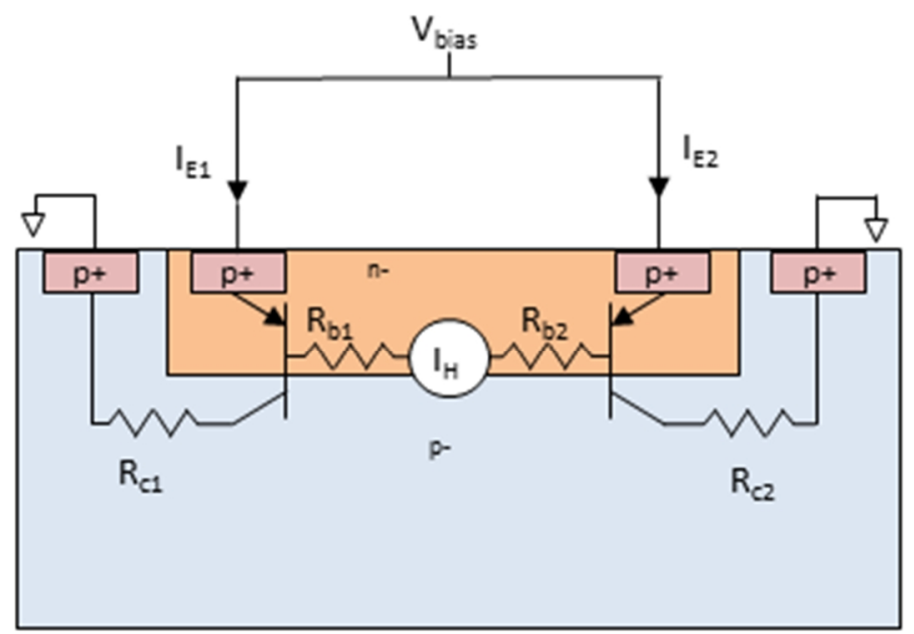

a)

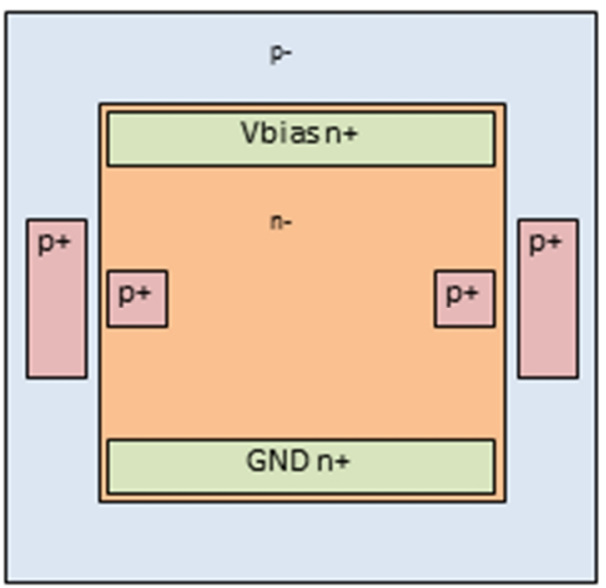

b)

Fig. 2 (a) Cross section and (b) top view of $n$-well Hall plate showing the presence of the parasitic vertical BJT between the $p$-type node $p+$ forming the emitter, the $n$-well $n$-forming the base and the substrate $p$ forming the collector. $I_{E}$ denotes the emitter current, $I_{H}$ the Hall current and $R_{b 1}, R_{b 2}, R_{c 1}$ and $R_{c 2}$ the relevant parasitic resistances

Fig. 3 shows a schematic representation of the Hall plate which can also be used as a first order simulation model. The Hall current $I_{H}$ is modelled as a current source whilst the Hall plate offset is modelled by adjusting $R_{d}$ which represents the mismatch that occurs in the plate resistance $R_{s 1}$ and $R_{s 2}$ and ultimately the zero magnetic field Hall current. Under ideal conditions, $R_{s 1}=R_{s 2}=R_{s}$. Typically in the technology used in this study, it was found that $R_{d}$ was in the order of $0.2 \%$ of $R_{S}$ which translates to approximately $2 \Omega$. $V_{\text {bias }}$ is used to set up the bias current $I_{\text {bias }}$ through the Hall plate causing a voltage drop of $V_{E B}$ across both $R_{S 1}$ resistors. For relatively low bias currents and magnetic fields, the voltages $V_{R s 1}$ and $V_{R S 2}$ across $R_{s 1}$ and $R_{S 2}$ will be equal and the base resistance will have a very small impact on the emitter Hall current. If however $V_{\text {bias }}$ increases to such extent that $V_{\text {bias }} / 2$ exceeds the forward voltage of the base emitter junction $V_{E B}$, the voltage across $R_{S 2}$ will increase at a faster rate than the voltage across $R_{S 1}$ and will become a source of non-linearity. Ideally $I_{B}$ should be kept very small, such that the current through $R_{s 1}$ and $R_{s 2}$ are approximately equal. $R_{c 1}$ and $R_{c 2}$ can be minimized through proper layout design of the BJT. Biasing the transistors requires that the base to emitter voltage $\left|V_{B E}\right|$ be greater than $0 \mathrm{~V}$ with a biasing voltage $V_{\text {bias }}$ across the Hall plate as given in Eq. ( 8 ) and thus:

$$
V_{\text {bias }} \approx 2 V_{B E} \approx 2 V_{R s 1} \approx 2 V_{R s 2} \text {. }
$$

Keeping $V_{\text {bias }}$ as low as possible also reduces the consumed power and hence also the Joule heating in the Hall plate and is one of the major advantages of this technique. This does however come at the expense of sensitivity. Fig. 4 shows both the pnp transistor's forward voltage $\left|V_{B E}\right|$ and gain $\beta$ versus the collector current $I_{C}$ characteristics as simulated from foundry data. It can be seen that a collector current as low as $1 \mathrm{nA}$ is sufficient to bias the transistor enough to get stable current gain. As the main aim was to illustrate a proof of concept, biasing the emitter at the same $V_{\text {bias }}$ greatly simplified the setup. For this experiment, this bias point is indicated in Fig. 4 by the dotted black line 
at approximately $2 \mathrm{~mA}$. From Fig. 4 it can also be seen that the ideal biasing conditions for the Hall device would be for a collector current between $1 \mathrm{nA}$ and $100 \mu \mathrm{A}$. This range yields a stable current gain factor Beta over many decades of collector current and will also yield a decrease in inter-chip variation as well as lower temperature dependencies.

Using the setup shown in Fig. 3 with $V_{\text {bias }}=1.7 \mathrm{~V}$, the Beta for each BJT was calculated by measuring the emitter and collector current separately. The base current and hence the BJT's Beta could be deduced.

Table 1 below shows the measured Betas for ten samples used in this experiment which consist of the average of both BJT's per plate. It can be seen that the inter-chip variation is relatively good, matching within approximately $\pm 2.5 \%$. This mismatch will however be larger between wafers and batches.

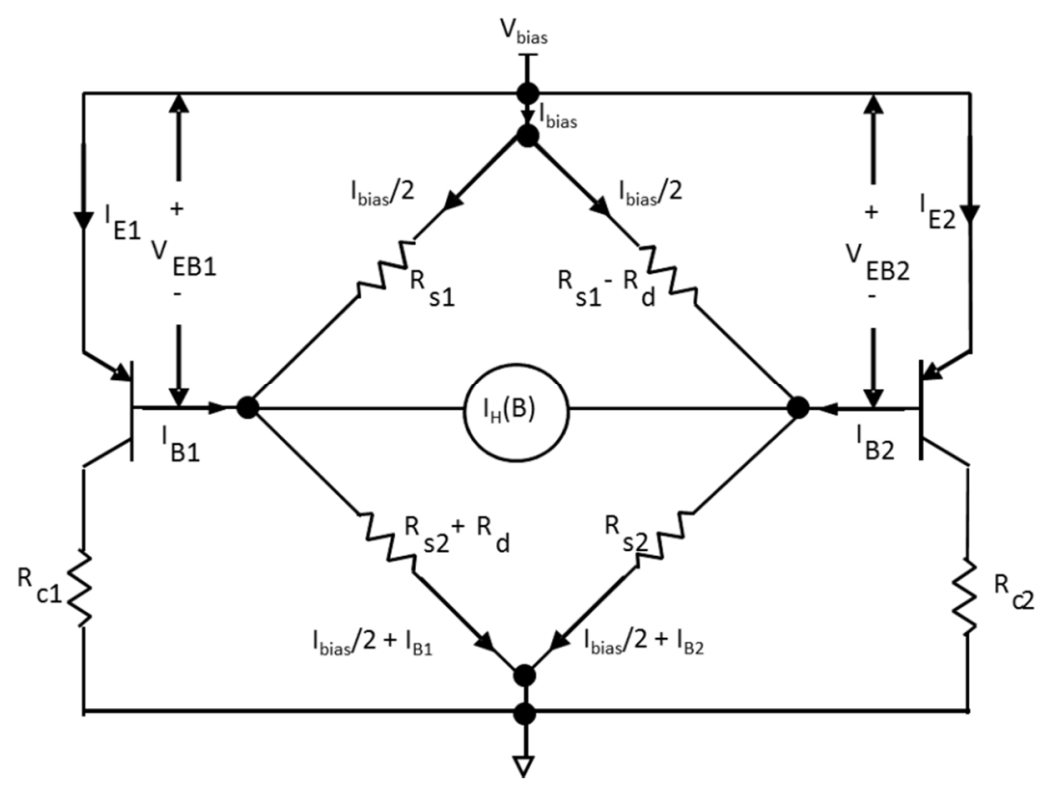

Fig. 3 Schematic representation of the Hall plate with integrated pnp transistors. $I_{E 1}$ and $I_{E 2}$ denotes the emitter current, $I_{B 1}$ and $I_{B 2}$ the base current, $I_{H}(B)$ the Hall current, $R_{C 1}$ and $R_{C 2}$ the collect parasitic resistances, $\boldsymbol{R}_{s 1}$ and $\boldsymbol{R}_{s 2}$ the Hall plate resistance, $\boldsymbol{R}_{d}$ the deviation component of the Hall plate resistance for modelling the offset effects, $V_{E B 1}$ and $V_{E B 2}$ the emitter to base voltages, $V_{\text {bias }}$ the bias voltage and $I_{\text {bias }}$ the resulting bias current through the Hall plate 
Table 1 Summary of the measured vertical BJT emitter current $I_{E}$ and collector current $I_{C}$ used to calculate the forward gain $\beta$ for a $V_{C E}$ of $1.7 \mathrm{~V}$

\begin{tabular}{lccc}
\hline Device number & $\boldsymbol{I}_{E}(\mathrm{~mA})$ & $\boldsymbol{I}_{\boldsymbol{C}}(\mathrm{mA})$ & Calculated $\boldsymbol{\beta}$ \\
\hline 1 & 2.251 & 1.688 & 2.998 \\
2 & 2.237 & 1.667 & 2.925 \\
3 & 2.218 & 1.653 & 2.926 \\
4 & 2.306 & 1.740 & 3.074 \\
5 & 2.269 & 1.702 & 3.002 \\
6 & 2.248 & 1.683 & 2.979 \\
7 & 2.244 & 1.678 & 2.965 \\
8 & 2.269 & 1.706 & 3.030 \\
9 & 2.234 & 1.669 & 2.954 \\
10 & 2.226 & 1.668 & 2.989 \\
Average & 2.250 & 1.685 & 2.984 \\
\hline \hline
\end{tabular}

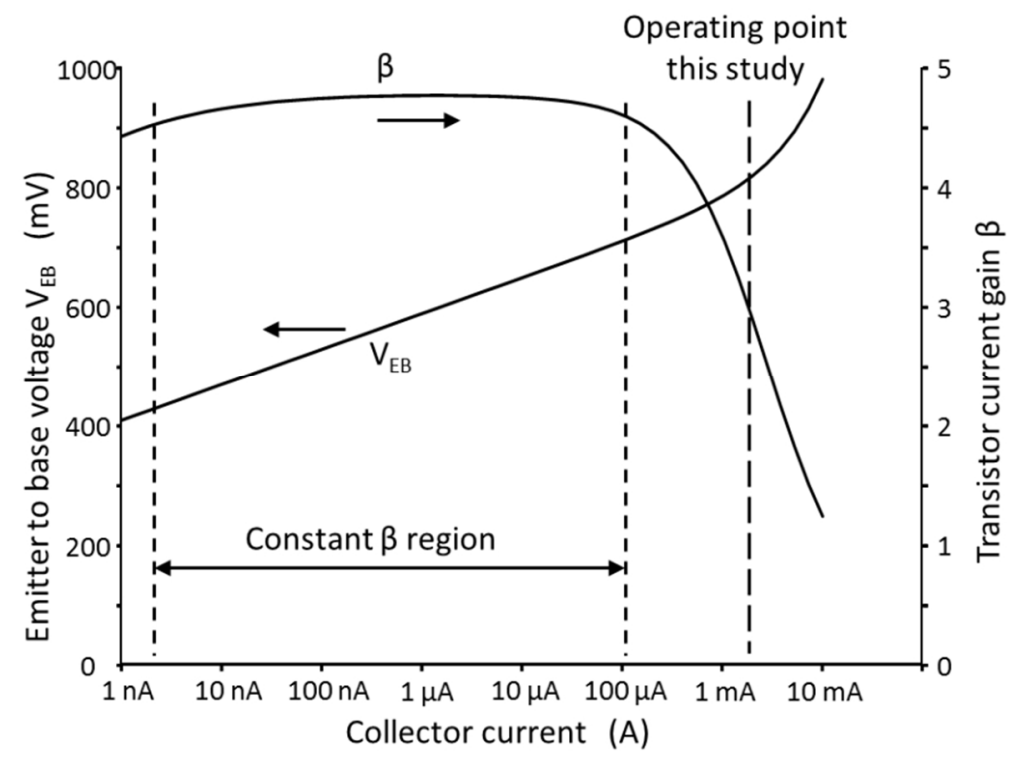

Fig. $4 \mathrm{BJT}$ forward current gain $\beta$ and forward base emitter voltage $V_{E B}$ as a function of the collector current $I_{C}$ for a similar sized BJT as modelled by the foundry

As we are most interested in how the emitter current translates into a measurable Hall effect parameter, it will be necessary to relate the emitter currents $I_{E 1}$ and $I_{E 2}$ to the effective Hall current $\Delta I_{E H}$ by measuring the difference between the emitter currents and is given by: 


$$
\Delta I_{E H}=I_{E 1}-I_{E 2}
$$

Rewriting $\Delta I_{E H}$ as a function of the base currents $I_{B 1}$ and $I_{B 2}$ results in:

$$
\Delta I_{E H}=(\beta+1)\left(I_{B 1}-I_{B 2}\right)
$$

with $\beta$ the forward current gain as defined in the technological electrical parameters. The potential gain this method offers is quite significant especially in more sophisticated technologies such as BiCMOS processes where a much higher $\beta$ may be achievable.

\section{Hall plate design}

\subsection{Hall effect characteristics}

A standard Hall plate with an area of $100 \mu \mathrm{m} \times 100 \mu \mathrm{m}$ was planned as a test structure based on the technological parameters of the $0.35 \mu \mathrm{m}$ CMOS technology. Substituting these technological parameters into Eq. ( 2 ) through ( 7 ), it was possible to determine the expected theoretical performance of the plate. A summary of the expected performance of the plate at the proposed bias voltage of $V_{\text {bias }}=1.7 \mathrm{~V}$ can be seen in Table 2 against which the Hall plate performance could be compared.

Table 2 Summary of calculated Hall plate parameters @ $V_{\text {bias }}=1.7 \mathrm{~V}$ based on CMOS technology specifications

\begin{tabular}{lcc}
\hline \hline Parameter & Calculated & Unit \\
\hline$B_{\max }$ & 185 & $\mathrm{mT}$ \\
$V_{H \max } @ B_{\max }$ & 23.24 & $\mathrm{mV}$ \\
$I_{H \max } @ B_{\max }$ & 26.56 & $\mu \mathrm{A}$ \\
$S_{A V}$ Hall voltage mode & 0.1256 & $\mathrm{VT}^{-1}$ \\
$S_{A l}$ Hall current mode & 0.1436 & $\mathrm{mAT}^{-1}$ \\
$S_{V}=S_{I}$ & 0.0739 & $\mathrm{~T}^{-1}$ \\
$G_{H}$ & 0.725 & - \\
$\theta_{\text {Hmax }}$ & 1.078 & $\mathrm{deg}$ \\
Plate width w & 100 & $\mu \mathrm{m}$ \\
Plate length I & 100 & $\mu \mathrm{m}$ \\
Plate thickness $t$ & 2 & $\mu \mathrm{m}$ \\
\hline \hline
\end{tabular}

A width to length ratio of unity was chosen as a practical device to use as it approximately coincides with the best trade-off between voltage or current sensitivity and the geometrical correction factor 
degradation [9]. According to [9], the maximum achievable voltage related sensitivity for a plate occurs for the shortest plate length. The mode of operation however changes from Hall voltage mode to Hall current mode once the plate becomes too short and thus for a very small Hall angle, the geometrical factor maximises at 0.742 . The maximum achievable voltage related sensitivity $S_{V \max }$ for Hall voltage mode or current related sensitivity $S_{\operatorname{Imax}}$ for current mode is thus $0.0756 \mathrm{~T}^{-1}$. The square Hall device is also a good choice as a test structure given it is very well documented in literature and simple to fabricate and study.

\section{Layout}

A layout was prepared according to the proposed design. The resulting Hall plate layout measuring $100 \mu \mathrm{m} \times 100 \mu \mathrm{m}$ is illustrated in Fig. 5 . The Hall device has $\mathrm{n}+$ ohmic contacts connected to $\pm V_{\text {bias }}$ through which the plate will be electrically biased. Normally the GND and $-V_{\text {bias }}$ nodes will be at the same potential, but for test purposes they were not connected on chip. The n-type contacts stretch across the entire width of the plate to ensure an evenly distributed current flow through the device. The $p+$ emitters are located at the midway point of the plate and are connected to $I_{E 1}$ and $I_{E 2}$ and each measure $19.6 \mu \mathrm{m} \times 8.8 \mu \mathrm{m}$. Finally a $\mathrm{p}+$ substrate contacts stretching around the entire plate are connected to the GND pad. Fig. 6 shows a photograph of the fabricated device.

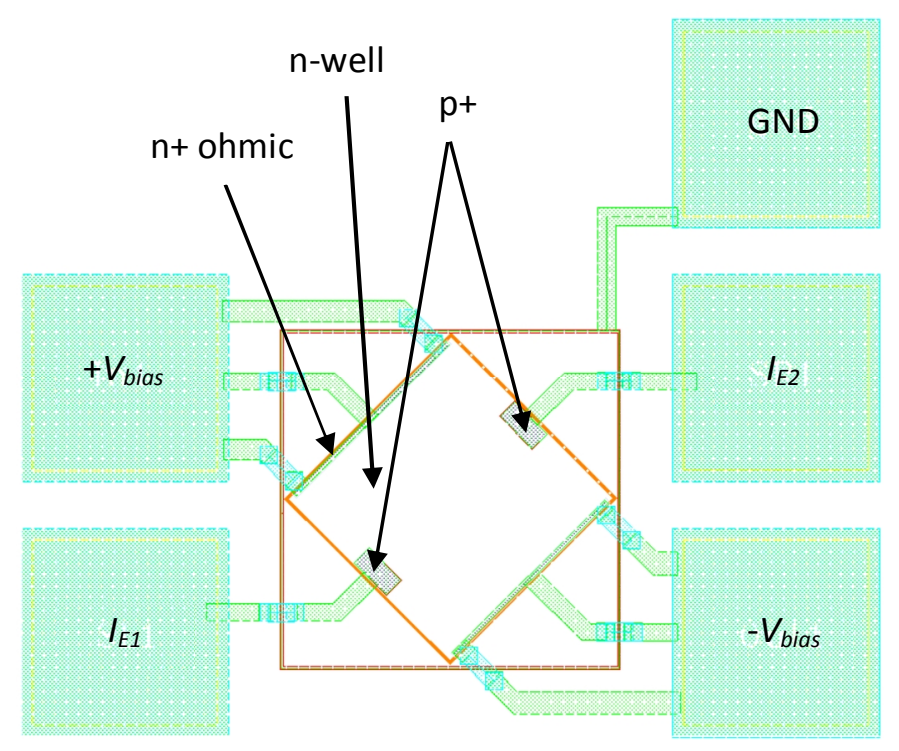

Fig. 5 Hall plate layout illustrating the biasing $\mathrm{n}+$ ohmic contacts $\pm V_{\text {bias, }}$, the $p+$ emitter contacts $I_{E 1}$ and $l_{E 22}$, the n-well Hall plate and the collector formed by the GND substrate contacts 


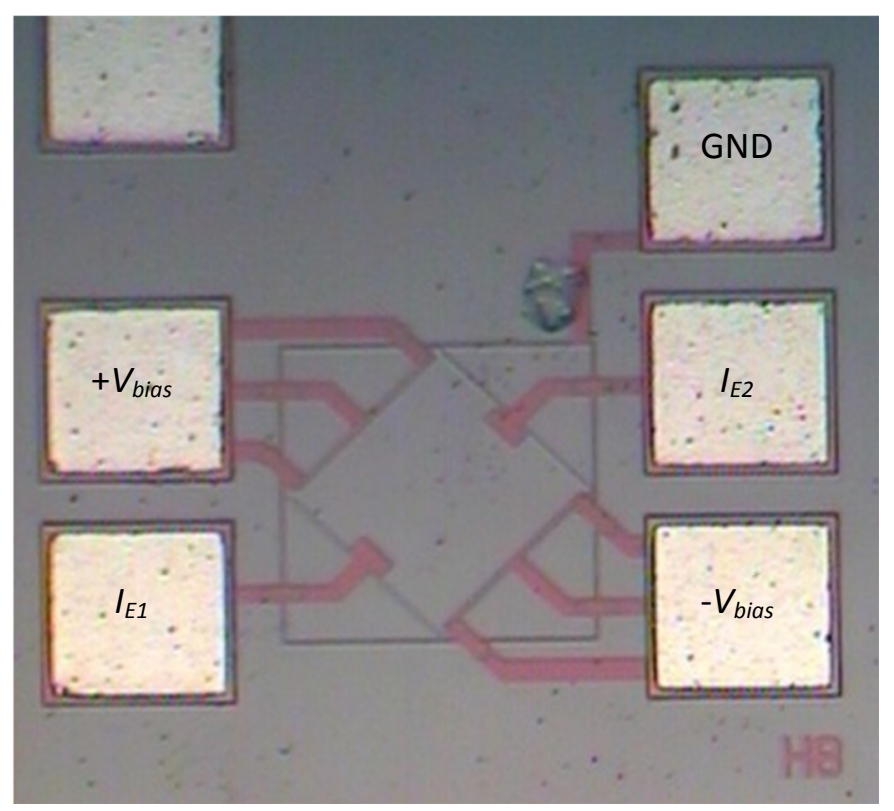

Fig. 6 Fabricated Hall plate

\section{Experimental procedure and results}

An A1302 linear ratiometric Hall sensor manufactured by Allegro was used as a reference for this experiment. The $\mathrm{A} 1302$ is a high precision linear Hall effect sensor that outputs a voltage that is directly proportional to the applied magnetic field. The $\mathrm{A} 1302$ was chosen as it has a very sensitive response of $1.3 \mathrm{mV} / \mathrm{G}$. At a supply of $5 \mathrm{~V}$, it provides for a very large dynamic range with high enough resolution for the intended aims of this study. Ten samples were manufactured and prepared for measurements. It was first required to measure the emitter and collector currents so that the active forward gain $\beta$ for each Hall plate's BJT could be calculated. This was done by using a bias voltage of $V_{\text {bias }}=+1.7 \mathrm{~V}$ applied to the Hall plate as well as the BJT emitters with ammeters between the emitters and the supply voltage. The calculated gains were averaged and the results reported in Table 3.

Table 3 Summary of BJT parameters at biased conditions as illustrated in Fig. 2 and Fig. 3

\begin{tabular}{lcc}
\hline Parameter & Measured & Unit \\
\hline$V_{\text {bias }}$ & 1.7 & $\mathrm{~V}$ \\
p-type area & $1.72 \mathrm{E}-6$ & $\mathrm{~cm}^{2}$ \\
$I_{C}$ & 1.67 & $\mathrm{~mA}$ \\
$I_{B}$ & 0.56 & $\mathrm{~mA}$ \\
$I_{E}$ & 2.23 & $\mathrm{~mA}$ \\
$\beta_{f}$ & 2.98 & \\
\hline \hline
\end{tabular}


Next the Hall plate resistances were measured such that the electron mobility for each individual sensor could be calculated. The measured results of the ten samples were then used to calculate the theoretical Hall current $I_{H}$ for each individual plate according to Eq. ( 3 ). Using the electrical design in Fig. 3, a physical setup was built upon which the Hall plate integrated circuit was mounted such that the magnetic field from a rare earth magnet directly above it would intercept the plate perpendicularly. The magnet was then moved up and down with linear magnetic field strength steps and the emitter currents measured using a MASTECH ${ }^{\circledR}$ MS8218 digital multimeter with a resolution of $1 \mu \mathrm{A}$ and hence also reflecting the uncertainty in the measurement. The measured differential emitter current thus consists of the differential base currents of the transistors multiplied by $\beta+1$ as indicated by Eq. ( 10 ). As with the Hall voltage, the Hall current appears positive through one of the BJT's and negative through the second, the sign reflecting the specific direction of the perpendicular field.

The differential emitter currents $\Delta I_{E H}=I_{E 2}-I_{E 1}$ for each device was measured individually. A comparison of a typical measured $\Delta I_{E H}$ current and the calculated elemental Hall currents $I_{H}$ is plotted in Fig. 7. The magnetic field itself was calculated by using the A1302 output voltage as reference. The Hall plate was subjected to a maximum magnetic field of approximately $\pm 185 \mathrm{mT}$ at a bias voltage of $V_{\text {bias }}=1.7 \mathrm{~V}$. From Fig. 7 it can be seen that a linear signal response is observed from the transistor circuit. The offset is in the order of $15 \mathrm{mT}$, which is not excessive, since no layout or circuit techniques were used to improve transistor matching. It can also be observed that the measured output current signal is significantly larger than the Hall current signal derived theoretically for the basic Hall element with no transistor readout. This is due to the current gain of the transistors.

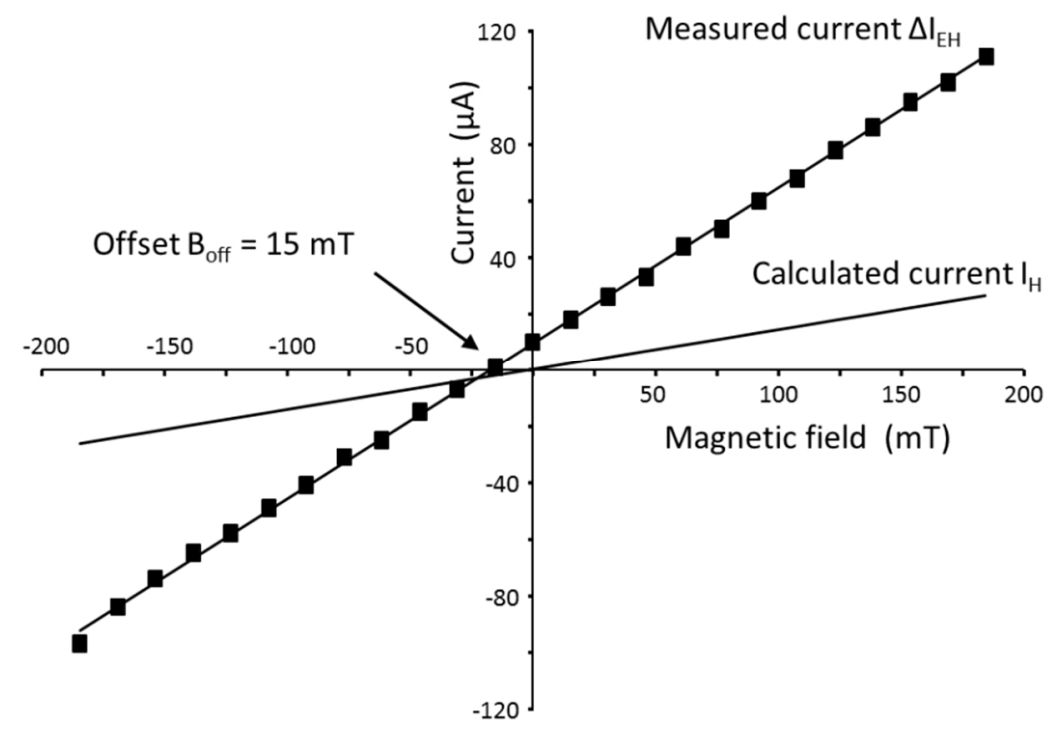

Fig. 7 Measured differential emitter current $\Delta I_{E H}$ of one Hall device compared to the theoretical Hall current $I_{H}$ in $\mu$ A versus the magnetic field $B$ in $\mathrm{mT}$ 
The average current gain of the ten BJT's was measured to be 2.98 which also compares well with the gain of 3 as illustrated by the simulation results in Fig. 4. It can thus be shown that the required sensitivity to measure 1 Gauss would be in the order of $150 \mathrm{nA}$, a feat easily achievable using very simple current mirroring techniques. Furthermore, by using high gain BJT's available in a BiCMOS process, significant gains can be made using this technique.

\section{Noise analysis}

Two noise sources are identified, namely $i_{n R}^{2}$, the noise contributed by the Hall resistive plate and $i_{n T}^{2}$, the input referred noise from the BJT.

The Hall plate noise $i_{n R}^{2}$ is dominated by its thermal noise and $1 / f$ flicker noise and can be approximated by Eq. ( 11 ) if recombination-generation noise is excluded:

$$
i_{n R}^{2}=S_{n R} \cdot \Delta f=\left[\frac{4 k T}{R_{S}}+\frac{\alpha}{n} \frac{1}{f} I^{2}\right] \Delta f
$$

Current $I$ is the bias current $I_{\text {bias }}$ through the device, $n$ the total number of charge carriers in the device, $\alpha$ is the Hooge parameter, a dimensionless value that may be associated with a specific device processing method and structure. Recently the Hooge parameter $\alpha \approx 10^{-5}$ has been determined for CMOS silicon Hall devices very similar to ours [20], and therefore we used this value in our calculations.

The input referred transistor noise $i_{n T}^{2}$ has shot noise components as well as a flicker noise component, as shown in Eq. ( 12 ). Since two transistors are connected to the Hall plate, the transistor noise contributions should be twice that of a single BJT.

$$
i_{n T}^{2}=S_{n T} \cdot \Delta f=2\left[2 q I_{B}+\frac{2 q I_{C}}{\beta^{2}}+\frac{K I_{B}}{f}\right] \Delta f
$$

The flicker coefficient $K$ will determine the corner frequency of the BJT noise spectral density where the flicker noise equals the "white" shot noise. Most of the BJT spectra measured in a detailed study had corner frequencies in the range of $10 \mathrm{~Hz}$ to $10 \mathrm{kHz}$ [21]. In our calculation a corner frequency of $1 \mathrm{kHz}$ was assumed, a typical value for BJT devices. 
The Hall noise current is $i_{n H}^{2}$ and will be given by:

$$
i_{n H}^{2}=S_{n H} \cdot \Delta f=i_{n R}^{2}+i_{n T}^{2}
$$

The relevant noise spectral densities as calculated for our device are shown in Fig. 8. From this figure it can be seen that at low frequencies the transistor $1 / f$ noise is slightly larger than the Hall plate noise. The $100 \mathrm{~Hz}$ frequency noise values are tabulated in Table 4. From the slopes in Fig. 7, the absolute device sensitivities $S_{A}$ could be determined for the plain Hall plate sensor, as well as the Hall sensor with BJT sensing devices.

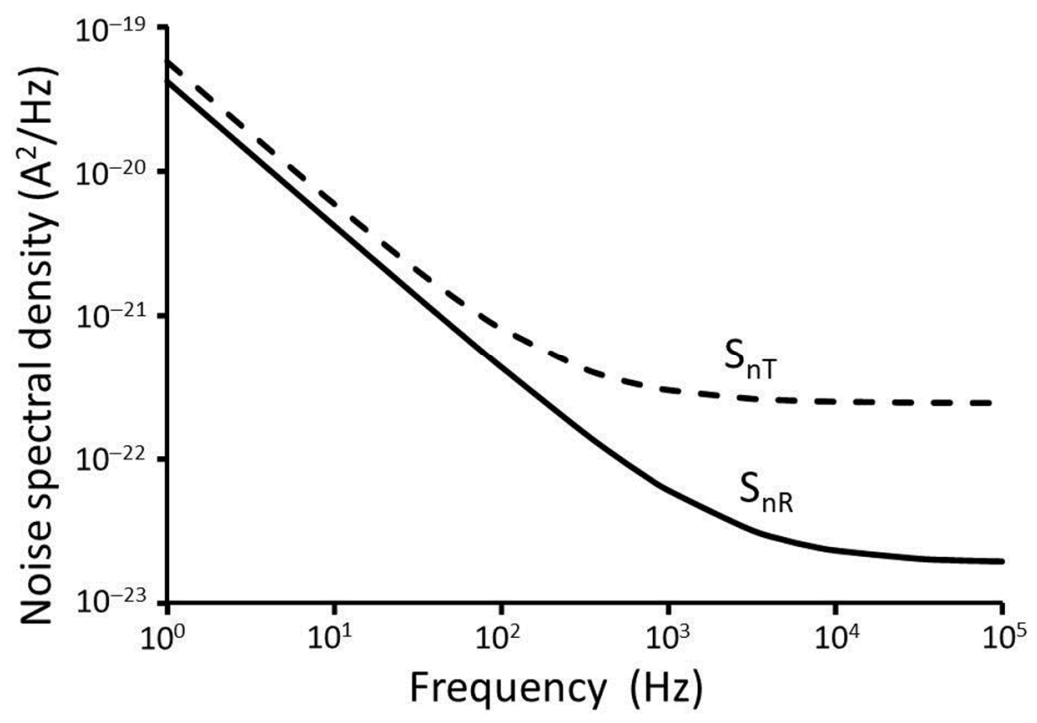

Fig. 8 Noise current spectral densities

Table 4 Summary of calculated noise contribution and minimum magnetic detection level at $100 \mathrm{~Hz}$ for i) a traditional plate without the integrated BJT's, and ii) the integrated BJT Hall device

\begin{tabular}{lccc}
\hline \hline & $\begin{array}{c}\text { Absolute } \\
\text { sensitivity } \mathrm{S}_{\mathrm{A}} \\
(\mathrm{A} / \mathrm{T})\end{array}$ & $\begin{array}{c}\text { Total input noise } \\
\text { density } \mathrm{S}_{\mathrm{nH}}\left(\mathrm{A}^{2} / \mathrm{Hz}\right)\end{array}$ & $\begin{array}{c}\text { Noise } \\
\text { equivalent field } \\
(\mathrm{T} / \sqrt{\mathrm{Hz}})\end{array}$ \\
\hline Hall plate & $140 \times 10^{-6}$ & $4.32 \times 10^{-22}$ & $0.15 \times 10^{-6}$ \\
Hall plate + BJT & $550 \times 10^{-6}$ & $12.4 \times 10^{-22}$ & $0.25 \times 10^{-6}$ \\
\hline \hline
\end{tabular}


From the above table the spot noise figure $N F=\left(S N R_{\text {out }}\right) /\left(S N R_{\text {in }}\right)$ of the BJT amplifier circuit can be determined as the ratio of the two input referred noise densities, giving us the value of $N F=4.6 \mathrm{~dB}$ at $100 \mathrm{~Hz}$ frequency. The noise figure can be lowered by reducing the BJT base current, although too low a base current will limit the dynamic range.

Measurements of CMOS Hall sensors with plate area $20 \mu \mathrm{m} \times 20 \mu \mathrm{m}=400 \mu \mathrm{m}^{2}$, layer thickness $1 \mu \mathrm{m}$ and drive current $0.2 \mathrm{~mA}$ resulted in a measured noise equivalent field of $5 \times 10^{-7} \mathrm{~T} / \mathrm{VHz}$ at $100 \mathrm{~Hz}$ [8]. The estimation of our Hall plate noise equivalent field is $1.5 \times 10^{-7} \mathrm{~T} / \sqrt{ } \mathrm{Hz}$ at $100 \mathrm{~Hz}$, which is in the same range.

Smaller area Hall plate devices will generate significantly higher $1 / f$ flicker noise than our quite large Hall plate. For $\mu$-Hall devices electron drift velocity saturation may also become one of the main limiting factors and for silicon this occurs at an electric field of approximately $30 \mathrm{kVcm}^{-1}$ [9]. For a bias voltage of $5 \mathrm{~V}$ for example, this corresponds to a minimum length of $1.5 \mu \mathrm{m}$. Scaling down the lateral dimensions, especially in present day deep submicron technologies, leads to an increase in noise on the Hall signal [8] and tends to dominate in smaller dimensions [11]. This is mainly due to the limited number of carriers available as well as other effects such as Joule heating. This makes the integrated BJT very suitable to integrate with $\mu$-Hall devices whereby much lower power consumption results in lower biasing currents and consequently $I^{2} R$ heating effects.

\section{Limitations}

\subsection{Layout}

As with the diode coupled BJT in a bandgap generator, care must be taken when currents flow through the substrate of an integrated device. The fundamental element that must be prevented in this case is latch-up. Latch-up occurs when adjacent structures form a pnpn structure that can be switched on when current flows through the substrate. By using well placed guard rails around the Hall plate, free carriers can be extracted from the substrate as soon as possible. The use of increased spacing to nearby devices is also a very effective method to reduce and even eliminate undesired latch-up scenarios. Typically an I/O should be able to withstand a minimum of $100 \mathrm{~mA}$ local substrate current according to the AEC-Q100-004 specification [22]. Using similar methods in our design should fairly easily ensure that substrate currents of a few $\mathrm{mA}$ can be tolerated.

\subsection{BJT Beta variation}

It is known that the current gain $\beta$ is a function of temperature, collector to emitter voltage $V_{C E}$ and collector current $I_{c}$. Fig. 9 illustrates this relationship using a standard foundry pnp BJT with similar area specifications. These variations in $\beta$ will translate into an error in the gain of the Hall current signal and will especially affect high accuracy linear applications negatively. For this reason it will be necessary to measure and adjust the gain of each BJT by either trimming the bias current through the BJT or by adjusting $V_{C E}$. For simple binary applications the variations in $\beta$ should be tolerable as 
the switching points can include enough margin such that this variation has negligible effect. This can be done through stimulating the Hall plate with a known magnetic field with a given bias condition and measuring the output. The bias condition can then be changed by either using an adjustable operational amplifier to vary $V_{C E}$ or by using adjustable current mirrors to vary the bias current through the Hall plate and implemented during wafer testing.

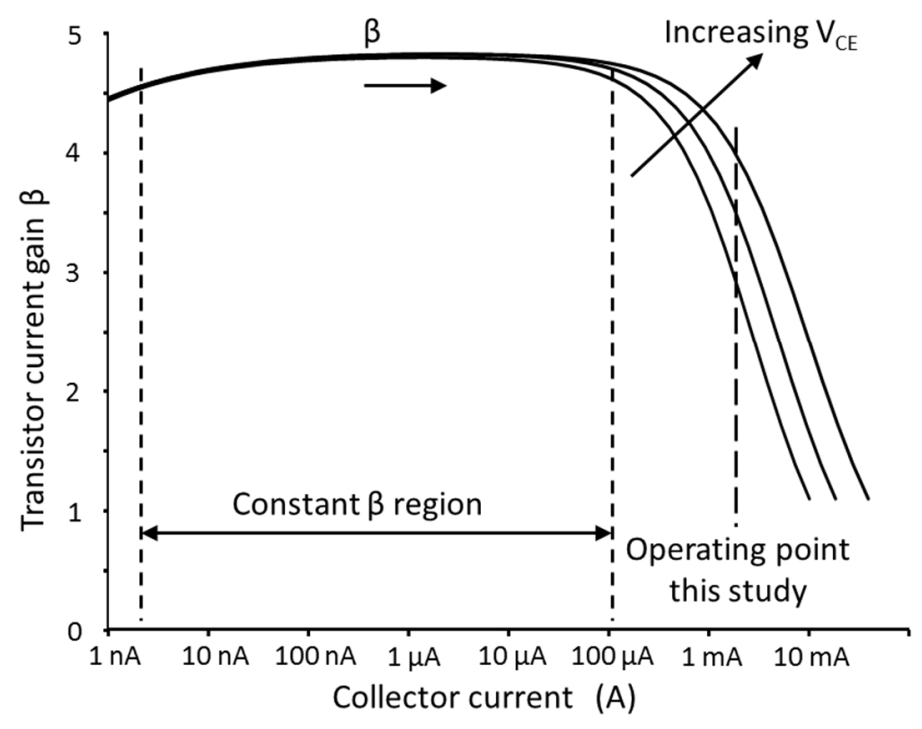

Fig. 9 BJT forward current gain $\beta$ as a function of the collector current $I_{C}$ as well as its dependency on the collector to emitter voltage $V_{C E}$ for a similar sized BJT as characterised by the foundry

\section{Conclusion}

A novel solution has been presented for the measurement of the Hall current in standard CMOS Hall plate devices. Using $\mathrm{p}$-type instead of $\mathrm{n}$-type contacts at the sensing location results in the activation of the vertical parasitic BJT's through which the Hall current can be measured by measuring the difference in emitter current flowing through each BJT. Correctly biasing the Hall device and BJT's, results in a signal gain of $\beta+1$ times the Hall current. The method is well suited to $\mu$-Hall devices to eliminate velocity saturation as well as to reduce Joule heating. The device was manufactured and shown to have very good correlation with the predicted theoretical results for a square Hall plate and also seems to be influenced by the geometrical factor in the same way traditional Hall voltage plates are. Future work will be focused on the design and implementation of a standalone sensor solution as well as dealing with the offset currents created by the biased BJT's. 


\section{References}

[1] R. S. Popovic, Hall Effect Devices-Magnetic Sensors and Characterization of Semiconductors, Bristol: Adam Hilger, 1991.

[2] J. Korvink and O. Paul, MEMS: A practical guide to design, analysis and applications, Norwich: William Andrew, Inc., 2006.

[3] C. Schott, J. Waser and R. S. Popovic, "Single-chip 3-D silicon Hall sensor," Sensors and Actuators A: Physical, vol. 82, pp. 167-173, 2000.

[4] M. Becherer, J. Kiermaier, S. Breitkreutz, G. Csaba, X. Ju, J. Rezgani, T. Kiesling, C. Yilmaz, P. Osswald, P. Lugli and D. Schmitt-Landsiedel, "On-chip Extraordinary Hall-effect sensors for characterization of nanomagnetic logic devices," Solid-State Electronics, vol. 54, pp. 1027-1032, 2010.

[5] K. Aledealat, G. Mihajlovic, K. Chen, M. Field, G. J. Sullivan, P. Xiong, P. B. Chase and S. von Molnar, "Dynamic micro-Hall detection of supermagnetic beads in a microfluidic channel," Journal of Magnetism and Magnetic Materials, vol. 322, pp. L69-L72, 2010.

[6] T. Q. Hung, S. Oh, J. Jeong and C. Kim, "Spin-valve planar Hall sensor for single bead detection," Sensors and Actuators A: Physical, vol. 157, pp. 42-46, 2010.

[7] J. Pascal, L. Hébrard, V. Frick, J. Kammerer and J. Blondé, "Intrinsic limits of the sensitivity of CMOS integrated vertical Hall devices," Sensors and Actuators A: Physical, vol. 152, pp. 21-28, 2009.

[8] K. Vervaeke, E. Simoen, G. Borghs and V. V. Moshchalkov, "Size dependence of microscopic Hall sensor detection limits," Review of Scientific Instrument,, vol. 80, pp. 1-7, 2009.

[9] R. S. Popovic, Hall Effect Devices, second ed., Bristol and Philadelphia: Institute of Physics Publishing, 2004, pp. 197-202;218-222;358.

[10] B. Razavi, Fundamental of Microelectronics, Wiley, 2008.

[11] G. Boero, M. Demierre, P. A. Besse and R. S. Popovic, "Micro-Hall devices: performance, technologies and applications," Sensors and Actuators A: Physical, vol. 106, pp. 314-320, 2003.

[12] E. W. Hall, "On a new action of the magnet on electric current," American Journal of Mathematics, vol. 2, no. 3, pp. 287-292, 1879.

[13] F. Riedijk and S. van Herwaarden, "Integration of micro-structures, micro-transducers and micro-electronics into micro-devices for space applications," in 3rd Round Table on Micro/Nanotechnologies for space, Noordwijk, 2000. 
[14] Z. B. Randjelovic, M. Kayal, R. S. Popovic and H. Blanchard, "Highly Sensitive Hall Magnetic Sensor Microsystem in CMOS Technology," IEE Journal of Solid-State Circuits, vol. 37, no. 2, pp. 151-159, 2002.

[15] J. E. Aubrey, G. Gubler, T. Henningsen and S. H. Koenig, "Piezoresistance and Piezo-Hall-Effect in n-Type Silicon," Physical Review, vol. 130, no. 5, pp. 1667-1670, 1963.

[16] A. Udo, "The piezo-Hall effect in n-silicon for arbitrary crystal orientation," in IEEE Sensors, Vienna, 2004.

[17] A. Udo, "The piezo-resistive effect in silicon for arbitrary crystal orientation," in IEEE Sensors, Vienna, 2004

[18] R. S. Popovic, Z. Randjelovic and D. Manic, "Integrated Hall-effect magnetic sensors," Sensors and Actuators A: Physical, vol. 91, pp. 46-50, 2001.

[19] W. E. Bulman, "Applications of the Hall Effect," Solid-State Electronics, vol. 9, pp. 361-372, 1966.

[20] K. Sun and M. Wang, "1/f Noise measurement and its doping dependancies of Si Hall sensors," in Physical and Failure Analysis of Integrated Circuits (IFPA), 2012 19th IEEE International Symposium, Singapore, 2-6 July 2012.

[21] T. Kleinpenning, "Low-Frequency Noise in Modern Bipolar Transistors: Impact of Intrinsic Transistor and Parasitic Series Resistances," IEEE Transactions on Electron Devices, vol. 41, no. 11, pp. 1981-1991, November 1994.

[22] IC latch-up test, Automotive Electronics Council, AEC-Q100-004-REV-D, 2012.

\section{Acknowledgement}

The authors would like to acknowledge Azoteq Pty(Ltd) for its financial support during this research.

\section{Biographies}

Dieter Mellet joined Azoteq Pty(Ltd) in 2003 from South African Micro-Electronic Systems (SAMES) where he was responsible for research and development of Hall Effect Sensors in CMOS for power metering systems. In 2012 he became en executive member of the board and is now responsible for the design and development of the next generation intelligent switches, proximity and touch devices as well as technology migration. He is one of the original developers of the capacitive proximity and touch technology and co-inventor of 1 international patent (USA and Europe). 
Mr. Mellet holds a Bachelors (Cum Laude) and Master's degrees in Electronic Engineering from the University of Pretoria and an MBA from the University of Stellenbosch Business School. He is currently studying for a Doctoral degree in Electronic Engineering at the University of Pretoria.

Monuko Du Plessis received the BEng, MEng and DEng degrees in electrical engineering from the University of Pretoria, Pretoria, South Africa in 1972, 1978 and 1984, respectively. He also received the BA (Psychology) and BCom (Hons) (Economics) degrees from the University of South Africa in 1989 and 1998 respectively.

He joined the Department of Electrical Engineering, University of Pretoria as Research Officer in the field of microelectronics and optoelectronics in 1973. He was the Director of the Carl and Emily Fuchs Institute for Microelectronics (CEFIM) at the University of Pretoria from 1991 to 2012, and is currently a Full Professor in the Department of Electrical, Electronic and Computer Engineering at the University of Pretoria. His current research interests are silicon photonics and analog CMOS integrated circuit design.

Prof. du Plessis is a member of SPIE and IEEE and a registered professional engineer with the Engineering Council of South Africa (ECSA). He has published widely in the field of microelectronics and related topics and is inventor of 11 international patents (USA and Europe) and several South African patents

Prof Du Plessis has published widely in the field of microelectronics and related topics and is coinventor of 13 international patents and several South African patents.

\section{Contact}

D.S. Mellet, tel: +27-21-863-0033; dieter.mellet@azoteq.com 